\title{
Increasing the Power Production of Vertical-Axis Wind-Turbine Farms Using Synergistic Clustering
}

\author{
Seyed Hossein Hezaveh ${ }^{1}$ (D) Elie Bou-Zeid ${ }^{1}$ (D) John Dabiri ${ }^{2} \cdot$ Matthias Kinzel $^{3}$. \\ Gerard Cortina $^{4} \cdot$ Luigi Martinelli ${ }^{5}$
}

Received: 14 June 2017 / Accepted: 4 June 2018 / Published online: 6 July 2018

(c) The Author(s) 2018

\begin{abstract}
Vertical-axis wind turbines (VAWTs) are being reconsidered as a complementary technology to the more widely used horizontal-axis wind turbines (HAWTs) due to their unique suitability for offshore deployments. In addition, field experiments have confirmed that vertical-axis wind turbines can interact synergistically to enhance the total power production when placed in close proximity. Here, we use an actuator line model in a large-eddy simulation to test novel VAWT farm configurations that exploit these synergistic interactions. We first design clusters with three turbines each that preserve the omni-directionality of vertical-axis wind turbines, and optimize the distance between the clustered turbines. We then configure farms based on clusters, rather than individual turbines. The simulations confirm that vertical-axis wind turbines have a positive influence on each other when packed in well-designed clusters: such configurations increase the power generation of a single turbine by about 10 percent. In addition, the cluster designs allow for closer turbine spacing resulting in about three times the number of turbines for a given land area compared to conventional configurations. Therefore, both the turbine and wind-farm efficiencies are improved, leading to a significant increase in the density of power production per unit land area.
\end{abstract}

Keywords Vertical-axis wind turbines · Wind energy · Wind farms · Wind-farm layout

Elie Bou-Zeid

ebouzeid@princeton.edu

1 Department of Civil and Environmental Engineering, Princeton University, Princeton, NJ 08540, USA

2 Department of Civil and Environmental Engineering, Department of Mechanical Engineering, Stanford University, Stanford, CA 94305, USA

3 Graduate Aerospace Laboratories, California Institute of Technology, Pasadena, CA 91125, USA

4 Department of Mechanical Engineering, The University of Utah, Salt Lake City, UT 84112, USA

5 Department of Mechanical and Aerospace Engineering, Princeton University, Princeton, NJ 08540, USA 


\section{Introduction}

Despite the concerted effort to improve energy efficiency and decouple economic growth from energy consumption, the U.S. Energy Information Administration projects that global total energy consumption will grow by about 45\% between 2015 and 2040 (U.S. Energy Information Administration 2013). Mitigating the concomitant large increase in greenhouse gas emissions necessitates exploring alternative lower-emission energy sources, particularly since the majority of the current fossil-based energy resources are finite and have other adverse side effects on the environment. Wind energy is expected to be one of the primary sources of clean, renewable energy that would allow a rapid transition away from fossil-fuelbased energy. In the USA, for example, wind power is projected to contribute around $20 \%$ of electrical energy by the year 2030 (Marquis et al. 2011). As a result, increasingly larger wind farms are being deployed, and the continued spread and expansion of these farms poses a challenge since the required land area will increase. A major goal of current research is thus to increase the wind-farm power density, i.e. how much energy can be produced per unit land area used.

In a wind farm, turbines should be far enough apart to allow wind speeds to recover, through lateral or vertical momentum entrainment, after deceleration by the upwind generator (Cortina et al. 2016). Spacing the turbines also reduces the fatigue load generated by turbulence from the upstream turbines and thus increases turbine lifetime (Chamorro and Porté-Agel 2009). The large majority of existing farms use horizontal-axis wind turbines (HAWTs); the behaviour of horizontal-axis wind turbines in large wind farms, and the required spacing between them, have been extensively studied (Wu and Porté-Agel 2012; Troldborg and Sørensen 2014). Calaf et al. (2010) investigated the vertical transport of momentum and kinetic energy in a fully-developed HAWT-array boundary layer (defined as the internal boundary layer developing above a wind farm). They showed that, for large wind farms, regeneration of the kinetic energy is mainly from downward vertical fluxes across the plane delineating the top of the farm, unlike farms with a limited number of windturbine rows where the streamwise advection of kinetic energy dominates. The concept of a wind-turbine-array boundary layer is particularly useful for wind farms where streamwise farm length is an order of magnitude larger than the depth of the atmospheric boundary layer (ABL) since the influence of such farms extends to the top of the ABL. Meyers and Meneveau (2010) used an actuator-disk model and large-eddy simulations (LES) to model large HAWT wind farms and investigate their interaction with the ABL. They have shown that a staggered wind farm can extract 5\% more power than an aligned configuration and in a follow-up study (Meyers and Meneveau 2012), investigated the optimization of turbine spacing in fully-developed wind farms. They showed that the ratio of land cost to turbine cost in the financial optimization analysis (maximizing power per unit cost) influences the deduced optimal spacing. Meyers and Meneveau (2012) indicate that the optimal turbine spacing is higher than that currently being used in HAWT wind farms. Recently, it has also been shown that the highest achievable mean wind-farm power is strongly dependent on the alignment of the turbine arrays relative to the mean wind direction, and the optimal alignment angle is significantly smaller than that in a perfectly-staggered farm (Stevens et al. 2014). For wind-farm sites with a dominant wind direction, these findings can be implemented to improve wind-farm performance.

All of the above, and other previous work, have focused on wind farms consisting of horizontal-axis wind turbines (Chamorro and Porté-Agel 2010; Lu and Porté-Agel 2011; Yuting 2011; Meyers and Meneveau 2012). However, recently Dabiri (2011) has suggested the 
possibility of an order of magnitude increase in power densities for wind farms when verticalaxis wind turbines (VAWTs) are used. Due to their axis of rotation, VAWT wakes and the flow in a VAWT farm are distinctly different from their HAWT counterparts. This potential increase in power density can be achieved by configuring VAWT farms with a closer spacing to better exploit the flow patterns created by upstream turbines. Dabiri (2011) and collaborators (Kinzel et al. 2012) performed experiments on various counter-rotating configurations of 9-m tall vertical-axis wind turbines and demonstrated that, unlike the typical performance reduction of horizontal-axis wind turbines with close spacing, there is an increase in VAWT performance when adjacent turbines are arranged to interact synergistically. However, high experimental costs and time requirements prevent the extension of these field investigations to large farm scales or the assessment of a large number of configurations. The previous findings thus only pertain to a limited number of turbines where the mean kinetic energy is primarily replenished by streamwise advection and cross-stream turbulent transport, rather than by vertical transport as in large farms. Our aim here is to bridge this research gap and assess the feasibility of increasing power density in large VAWT farms using a synergistic clustering of turbines. Building on Hezaveh et al. (2016), where a LES model for verticalaxis wind turbines was extensively validated and the flow recovery in the wake of a single turbine investigated, here we simulate the interactions of multiple vertical-axis wind turbines in small clusters, and subsequently use these clusters to design large VAWT farms.

\section{Numerical Model}

In order to investigate vertical-axis wind turbines in the ABL, we used the LES model with a VAWT actuator-line model (ALM-LES) presented and validated in Hezaveh et al. (2016), and present here a brief overview only. In this model, which is a variant of a LES model that has been previously used and validated for flow around horizontal-axis wind turbines (Chamorro and Porté-Agel 2009; Calaf et al. 2010, 2014; Lu and Porté-Agel 2011) and other complex flows (Bou-Zeid et al. 2007; Huang and Bou-Zeid 2013; Li et al. 2016), the following continuity and Navier-Stokes equations are solved at each timestep for the large resolved scales assuming an incompressible flow with a mean in vertical hydrostatic equilibrium

$$
\begin{gathered}
\frac{\partial \tilde{u}_{i}}{\partial x_{i}}=0, \\
\frac{\partial \tilde{u}_{i}}{\partial t}+\tilde{u}_{j}\left(\frac{\partial \tilde{u}_{i}}{\partial x_{j}}-\frac{\partial \tilde{u}_{j}}{\partial x_{i}}\right)=-\frac{1}{\rho} \frac{\partial \tilde{p}^{*}}{\partial x_{i}}-\frac{\partial \tau_{i j}}{\partial x_{j}}+F_{i}+F_{i}^{t},
\end{gathered}
$$

where $\tilde{u}_{i}$ is the resolved velocity vector with the tilde denoting a filtered quantity, $(u, v, w)$ are its streamwise, cross-stream and vertical components, respectively. This instantaneous velocity is decomposed into a mean $U_{i}$ and a resolved perturbation $u_{i}^{\prime} ; x_{i}$ is the position vector with components $(x, y, z)$ in the streamwise, cross-stream and vertical directions respectively, $\tilde{p}^{*}$ is a modified pressure that includes the resolved and subgrid-scale turbulent kinetic energies, $\rho$ is the air density, $F_{i}$ is the mean pressure gradient driving the flow, $\tau_{i j}$ is the deviatoric subgrid-scale stress tensor; and $F_{i}^{t}$ represents the aerodynamic forces of the turbine blades on the airflow. Note the omission of the Coriolis force, which is assumed to have no significant impact at such small distances (about $10 \mathrm{~m}$ ) from the Earth's surface. At each timestep, $F_{i}^{t}$ is computed using the actuator-line model as detailed in Hezaveh et al. (2016). The horizontal boundary conditions are numerically periodic, but non-periodic flows 


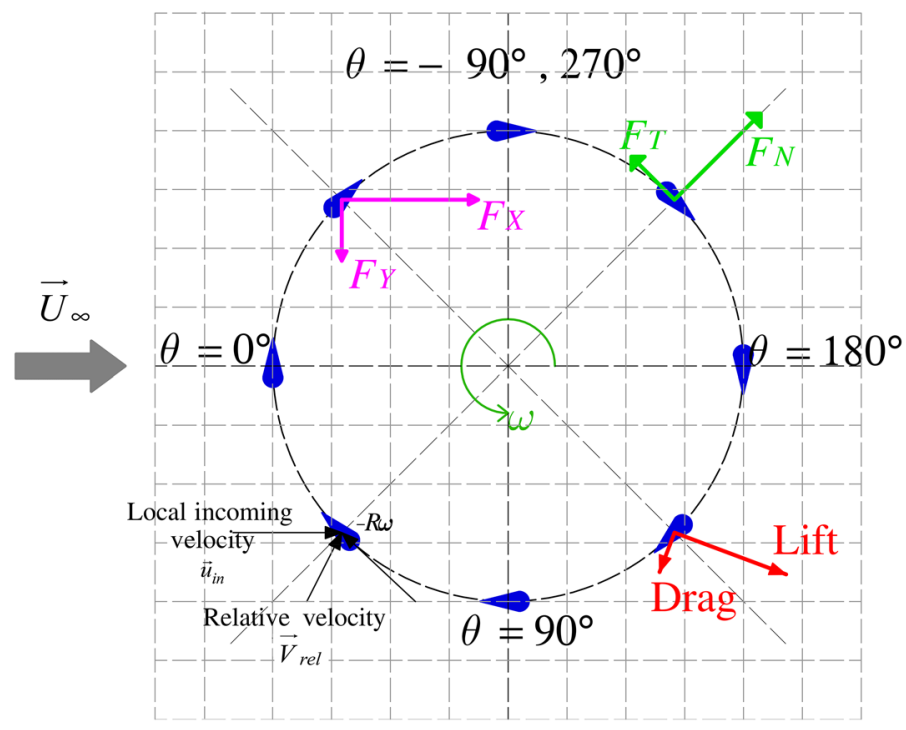

Fig. 1 Schematic two-dimensional cross-section (top view) of the VAWT blade path, the forces on the blades, and representative LES grid cells. $\theta$ is the azimuthal angle denoting the angular location of the blade relative to the incoming flow direction (defined positive in the same direction as the turbine rotation); it continuously varies in time for each blade as the turbine rotates. The depicted relative scales of the blade chord length to the LES grid cell illustrate that they are comparable, but their exact ratio (might be larger or smaller than 1) varies in the different simulations and the figure is not to scale. Adapted from Hezaveh et al. (2016)

can be simulated using an inlet sponge region as shown later. At the top boundary, zero vertical velocity and zero shear stress are imposed. The bottom boundary has zero vertical velocity, while the surface shear stress is imposed using an equilibrium logarithmic-law wall model with a wall roughness length $z_{0}=10^{-6} z_{i}$, where $z_{i}$ is the depth of the computational domain used to normalize all length scales in the model $\left(z_{i}=25 \mathrm{~m}\right)$. The details of the wall and subgrid-scale models are provided in Bou-Zeid et al. (2005). The model details are summarized in Fig. 1: an angle of attack ( $\alpha$, the angle between the blade chord and the flow velocity relative to the blade $\vec{V}$ rel) is first computed by knowing the location of each blade represented as a vertical line in the actuator-line model, the upstream undisturbed flow velocity $\left(\vec{U}_{\infty}\right)$, and the rotational speed of the turbine $(\omega)$. This then allows us to obtain the lift and drag force coefficients, $C_{L}$ and $C_{D}$ respectively, to be calculated from experimental data, blade-resolving Reynolds-averaged simulations, or tabulated airfoil data after applying a dynamic stall correction, as in Hezaveh et al. (2016). $C_{L}$ and $C_{D}$ are then used to compute the normal and tangential force coefficients, $C_{N}$ and $C_{T}$ respectively,

$$
\begin{aligned}
& C_{N}=\left|C_{L}\right| \cos \alpha+\left|C_{D}\right| \sin \alpha, \\
& C_{T}=\left|C_{L}\right| \sin \alpha-\left|C_{D}\right| \cos \alpha,
\end{aligned}
$$

which are then used to compute the corresponding forces

$$
\begin{aligned}
\mathrm{d} F_{N}(\theta) & =\frac{1}{2} \rho c V_{\mathrm{rel}}^{2} C_{N} \mathrm{~d} z, \\
\mathrm{~d} F_{T}(\theta) & =\frac{1}{2} \rho c V_{\mathrm{rel}}^{2} C_{\mathrm{T}} \mathrm{d} z,
\end{aligned}
$$




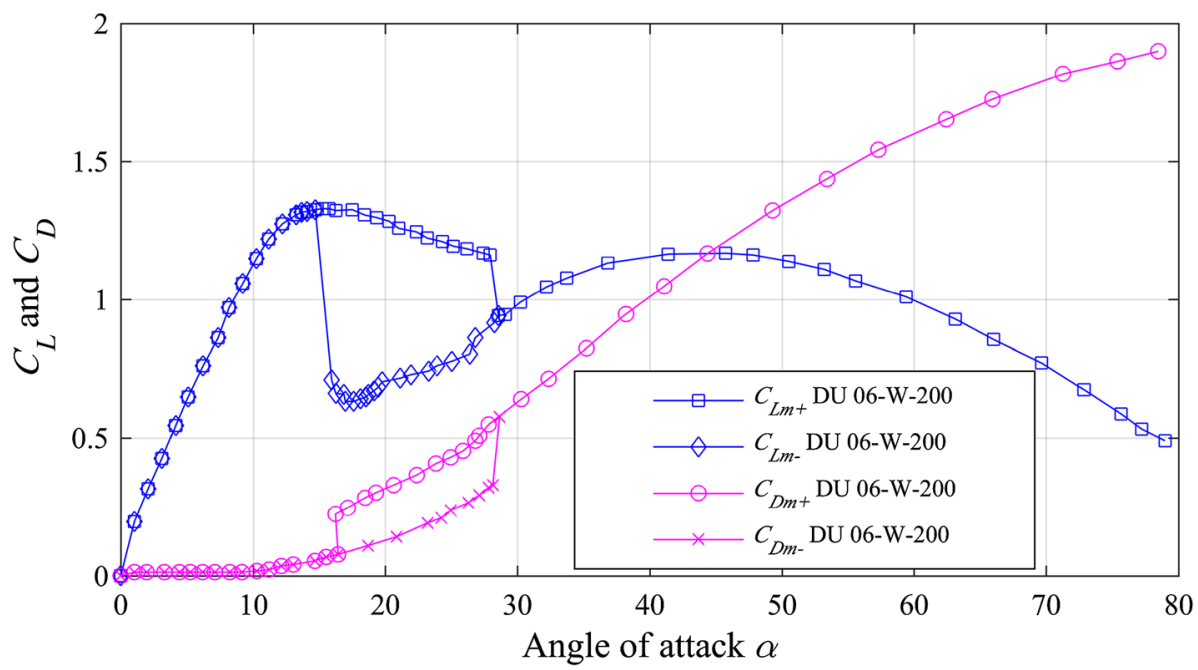

Fig. 2 Dynamic $C_{L}$ and $C_{D}$ for the DU 06-W-200 blade type as measured by (Claessens 2006). The + subscript is for $\mathrm{d} \alpha / \mathrm{d} t>0$ and the - subscript is for $\mathrm{d} \alpha / \mathrm{d} t<0 ; \mathrm{d} \alpha / \mathrm{d} t$ is the time rate of change of the angle of attack

where $c$ is the blade chord length, $\theta$ is the azimuthal angle of the blade at a given time, and $\mathrm{d} z$ is the vertical length of blade occupying each LES grid cell. Finally, the forces in the LES Cartesian coordinate system are obtained as,

$$
\begin{gathered}
\mathrm{d} F_{x}=\mathrm{d} F_{N}(\theta) \cos \theta+\mathrm{d} F_{T}(\theta) \sin \theta, \\
\mathrm{d} F_{y}=\mathrm{d} F_{N}(\theta) \sin \theta-\mathrm{d} F_{T}(\theta) \cos \theta .
\end{gathered}
$$

In order to evaluate $C_{L}$ and $C_{D}$ herein, actual dynamic curves for lift and drag forces determined experimentally were implemented into the numerical model. Since we aim to mimic the turbines used in the field experiments for vertical-axis wind turbines reported by Dabiri (2011) and Kinzel et al. (2012), we simulate the same DU-06-W-200 airfoil. Claessens (2006) has conducted several numerical and wind-tunnel experiments at various Reynolds numbers and tabulated the lift and drag curves for different airfoil blade types. Figure 2 depicts their measured values for the DU-06-W-200 airfoil, which include the dynamic-stall effect (directly accounted for in the wind-tunnel measurements). Based on the sign of $\mathrm{d} \alpha / \mathrm{d} t$, the instantaneous rate of change of the angle of attack, different paths emerge in these curves in Fig. 2: $C_{L m+}$ versus $C_{L m-}$ and $C_{D m+}$ versus $C_{D m-}$. We note that the DU-06-W-200 blade type is redesigned from the NACA 0018 airfoil and is $2 \%$ thicker, and cambered rather than symmetric (Claessens 2006). This blade type and the experimental results reported in Fig. 2 are used throughout.

\section{Results and Discussions}

\subsection{Validation Against Field Measurements}

As mentioned before, Dabiri (2011) and Kinzel et al. (2015) report on several field measurements with different configurations of 9-m tall vertical-axis wind turbines using the same blade type and rotor configuration we adopt here. They have shown that, by using 
Table 1 Turbine characteristics from Dabiri (2011) and Kinzel et al. (2015)

\begin{tabular}{lll}
\hline Variable & Symbol (units) & Value \\
\hline $\begin{array}{l}\text { Number of turbines } \\
\begin{array}{l}\text { Number of blades per } \\
\text { turbine }\end{array}\end{array}$ & $n$ & 2 \\
Rotor diameter & $D(\mathrm{~m})$ & 3 \\
Blade vertical length & $z_{t}(\mathrm{~m})$ & 1.2 \\
Blade chord length & $c(\mathrm{~m})$ & 6.1 \\
Airfoil section type & - & 0.11 \\
Solidity & $N c / \pi D$ & $\mathrm{DU} 06-\mathrm{W}-200$ \\
Tip-speed ratio & $\lambda$ & 0.275 \\
$\quad$ (selected at & & 2.18 \\
maximum $\left.C_{P}\right)$ & & \\
\hline
\end{tabular}

counter rotating vertical-axis wind turbines and special configurations, the turbines can exploit the flow deflection from upwind adjacent turbines and there is a potential of a one order-of-magnitude increase in power density. To complement the previous validation of this ALM-LES model performed against laboratory experiments (Hezaveh et al. 2016), and to ensure that the simulations accurately represent the flow in between multiple turbines and therefore within and in the wake of turbine clusters, we compare our LES results to the fieldmeasured data described in Kinzel et al. (2015) for two adjacent counter-rotating turbines. This is the first validation of our model against data from real-sized VAWT field measurements and, to the best of our knowledge, the first validation of any vertical-axis wind turbine ALM-LES against field data.

The details of the experimental set-up are presented in Table 1, and the schematic configuration is shown in Fig. 3. The 1200-W turbines are a modified version of a commercially available model from Windspire Energy Inc. (Dabiri 2011) and they were placed 1.6D apart ( $D$ is the rotor diameter). The velocity profiles were measured at 16 points with streamwise coordinates (relative to the line joining the centre of the turbines) of $x=-15 D,-1.5 D, 2 D$, and $8 D$ and elevations above ground of $z=3,5,7$ and $9 \mathrm{~m}$. All velocity components are normalized using a measured $10-\mathrm{m}$ wind speed from a meteorological tower in the vicinity of the experiments (Araya et al. 2014; Kinzel et al. 2015), which took place in Antelope Valley, north of Los Angeles, California. Further details about the measurements are provided in Appendix A-also see Dabiri (2011) for further information.

The computational domain has $L_{x} \times L_{y} \times L_{z}=31.2 \mathrm{~m} \times 15.6 \mathrm{~m} \times 25 \mathrm{~m}$, respectively spanned by $128 \times 64 \times 192$ grid nodes. This resolution yields about $5 \times 5$ horizontal grid points spanning each turbine rotor (five in each direction). The distance between the domain inflow and turbines was set equal to the distance between the furthest upstream measurement point and the turbines in the experiment, that is $15 \mathrm{D}$. In order to match the inflow conditions such as turbulence intensity and mean upstream wind speed profile in the LES to the observed field data, a precursor periodic simulation was run to generate the inflow. The roughness length and friction velocity of this precursor simulation were calibrated (with adopted values of $0.001 \mathrm{~m}$ and $0.5 \mathrm{~m} \mathrm{~s}^{-1}$, respectively) to yield the experimentally-observed logarithmic velocity profile measured $15 D$ upstream of the turbines. The inflow and validation simulations were conducted with neutral stability and, as detailed later, field experimental periods were selected during near-neutral conditions. Then, $y-z$ slices of instantaneous velocity and pressure were saved at each timestep and fed to the simulation with the turbines as upwind-inflow boundary conditions. 


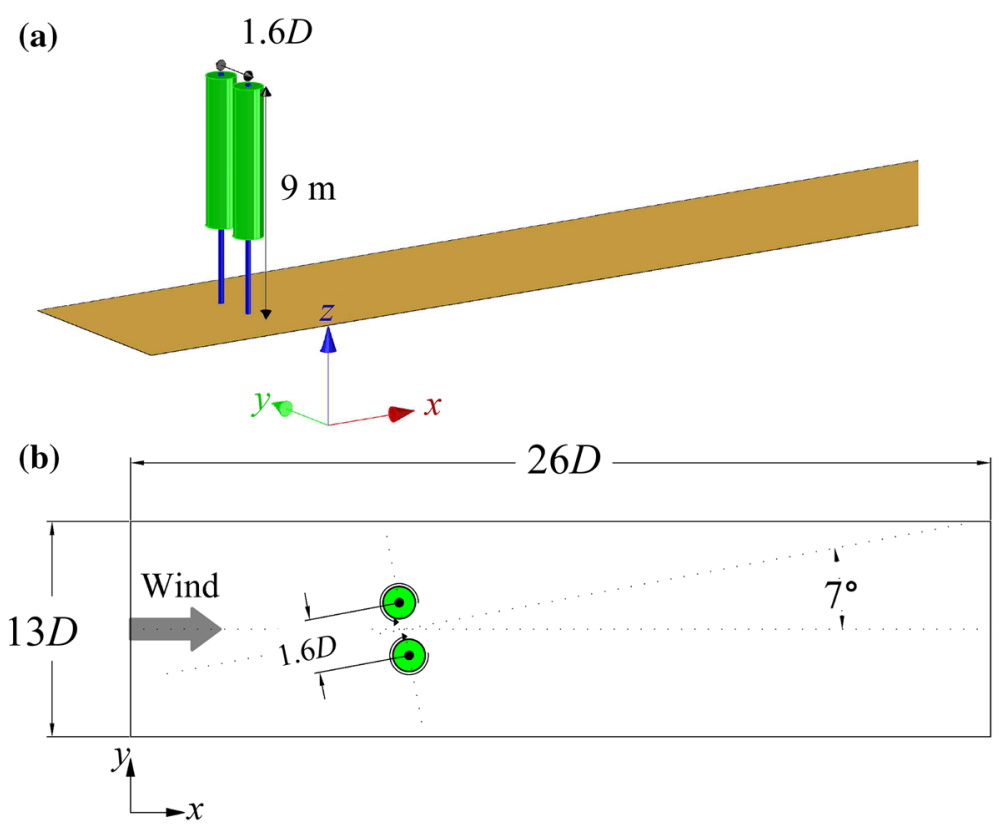

Fig. 3 Schematic of a the two turbines in the three-dimensional flow domain, and $\mathbf{b}$ top view of the computational domain
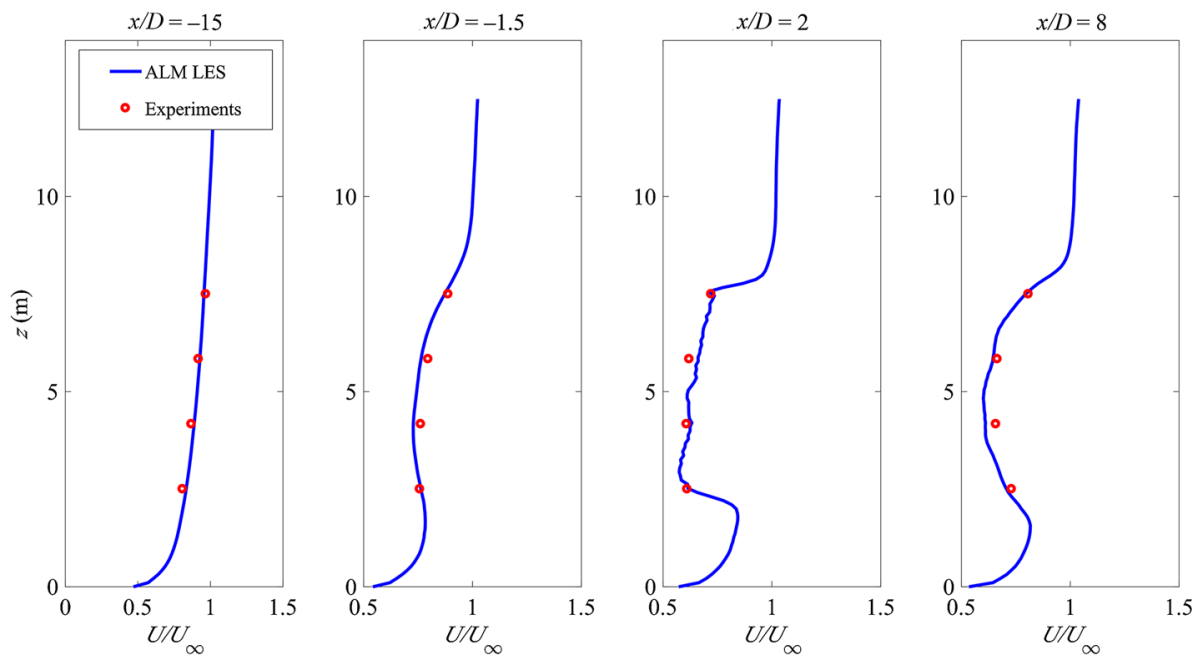

Fig. 4 Profiles of incoming and wake wind speed for the ALM-LES model versus field experimental data

The results are shown in Fig. 4, and it is clear that the ALM-LES model is capable of closely reproducing the wake generated by the interactions of the two counter-rotating vertical-axis wind turbines (the blades move towards the back when facing the other turbine so that the flow acceleration in between the two rotors is maximized). We should emphasize that it is essential to provide the LES with accurate inflow (left panel of Fig. 4, from the precursor simulation) for the experimental profiles near and behind the turbines (right three 
panels of Fig. 4) to be reproduced accurately. These results confirm that the ALM-LES produces realistic wakes even where turbines are interacting, and hence the model can be used to investigate large wind farms and VAWT clusters with confidence. It should also be mentioned that the ALM-LES model is capable of realistically capturing wake meandering, but this meandering does not appear in the figures herein since we only show mean velocities. Furthermore, the omission of the Coriolis force does not influence the result given the high Rossby number in the atmospheric surface layer at such low elevations. While the Coriolis force induces Ekman turning, for the omni-directional vertical-axis wind turbines the effect on performance is smaller than for horizontal-axis wind turbines.

\subsection{Vertical-Axis Wind Turbine Cluster Design: Geometric and Shading Considerations}

Clustering vertical-axis wind turbines in small arrangements have been shown to have several advantageous implications for power generation (Dabiri 2011). The global performance of the turbines is enhanced since the downstream turbines benefit from the flow-deflection effect and the resulting higher flow speed induced by upstream turbines. However, depending on the wind direction relative to the alignment of the turbine arrays in the farm, compact clustering might also have negative effects when one turbine is mainly in the wake/shadow of an upstream rotor. For example, if two turbines are clustered together, the range of wind directions for which one of the turbines is in the shadow (partially or fully) of the other is $2 \beta$, where $\beta=\tan ^{-1}(2 D / 2 L)$ (Fig. 5, left), $L$ being the turbine spacing (centre to centre) in a cluster. We note that this is a purely geometric consideration that does not account for the expansion of the wake. On the other hand, when the flow is approximately perpendicular to the centre-to-centre axis, the higher induced speed in-between the two turbines is not being exploited.

By introducing one additional turbine, the range of wind directions where two turbines can directly shadow each other is increased to $6 \beta$ (Fig. 5, middle). However, the third turbine can benefit from the higher wind speed induced in-between the two upstream turbines or the two downstream rotors can benefit from the transverse flow deflection of the upstream turbine (depending on wind direction). This has the potential to result in power production from these three turbines that is greater than the power from three distant non-interacting ones (this improvement depends on $L / D$, as shown below). By increasing the number of turbines in
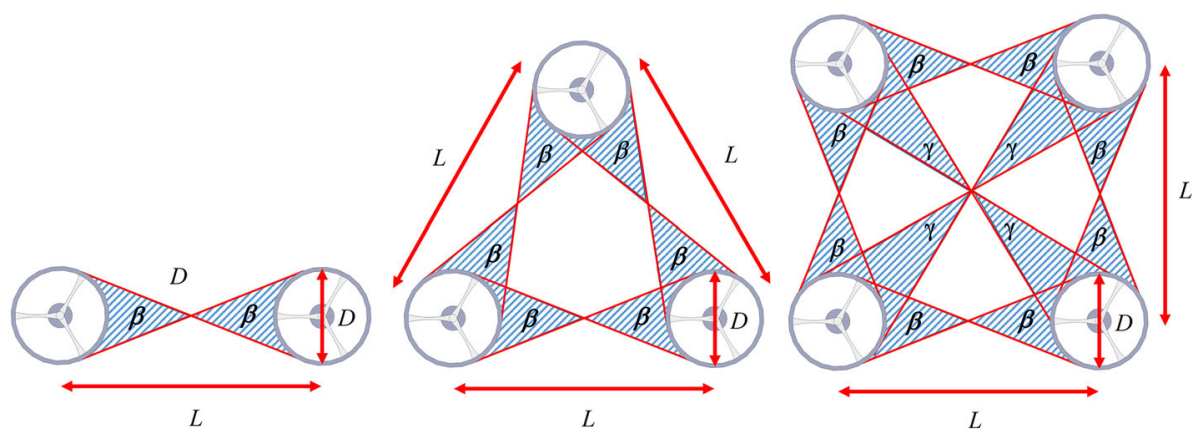

Fig. 5 Wind directions in which vertical-axis wind turbines are in the wake of an upstream rotor for two, three and four turbines $(\gamma \approx \beta)$ 


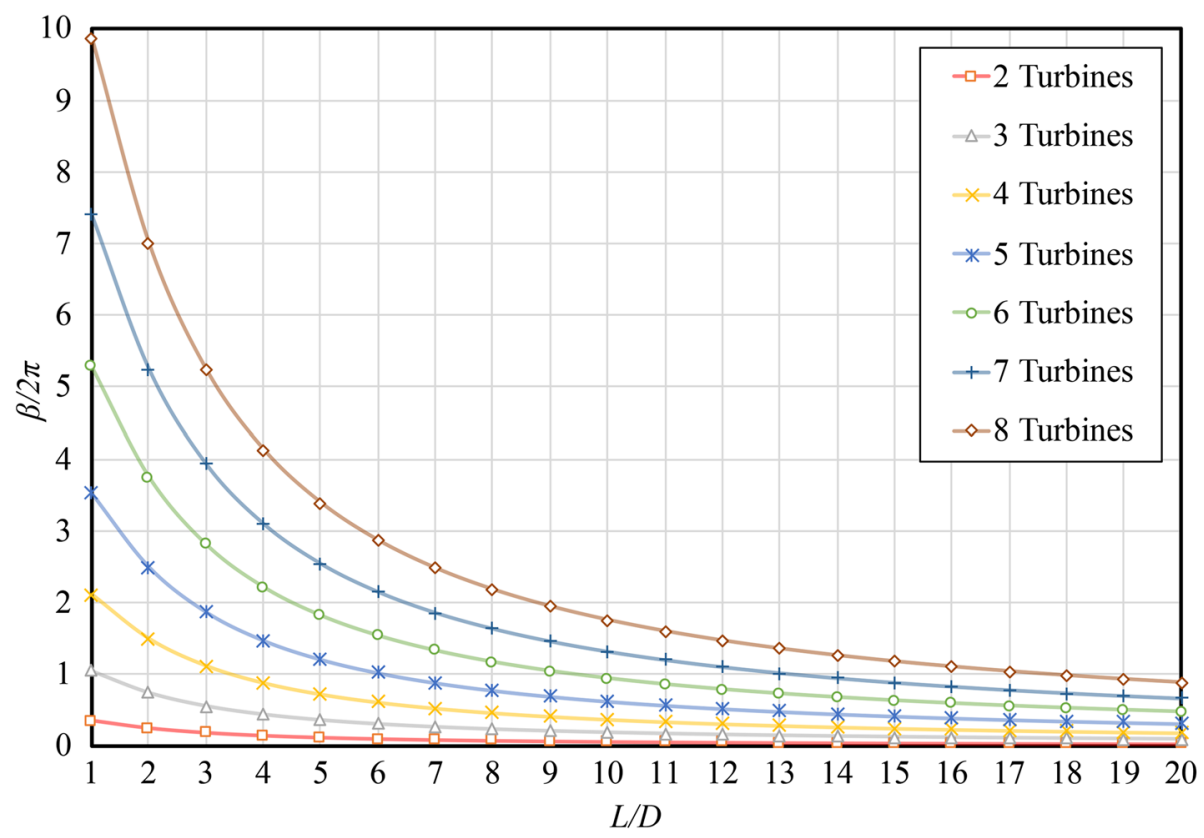

Fig. 6 Variation of $\beta$, the cumulative shadowing angle, with the $L / D$ ratio and the number of turbines in a cluster $n$

the cluster beyond three, the flow-related benefits decrease and the range of wind directions where the turbines shadow each other increases to $n(n-1) \beta$, where $n$ is the number of turbines in the cluster (e.g., Fig. 5, right). In Fig. 6, the variation of $\beta$ with $L / D$ and $n$ for various clusters is shown; physically, $\beta$ represents the total range of wind directions where shadowing influences the turbines. A value of $\beta=\pi$ implies, for example, that one turbine is partially or fully shadowed for $50 \%$ of possible wind directions, or alternatively that two turbines are shadowed for $25 \%$ of possible wind directions. As such, $\beta$ is the cumulative (partial or full) shadowing of all turbines from all possible wind directions and it can therefore exceed $2 \pi$. By increasing the value of $L / D$ of a cluster, $\beta$ is reduced, while on the other hand, increasing $n$ results in higher $\beta$. For $n>3$, the $\beta / 2 \pi$ value can become larger than 1 , which indicates that there is no wind direction for which the turbines are not casting at least partial shadows on each other. However, one notes that, for $L / D>5$, the differences between the $\beta$ values for $n=2$ and $n=3$ are minor. Moreover, a clustering with higher $n$ has the important benefit of using a smaller land area. Therefore, the most efficient design for a cluster when there is no dominant wind direction at the site seems to be a triangle $(n=3)$ since it has a limited $\beta$, while at the same time allowing for compact clustering and synergistic interaction between the turbines. A value of $n=4$ almost doubles the shadowing angle $\beta$, with no increase in the wind-direction range for which synergistic interactions occur. Therefore, we focus on triangular clusters hereafter.

\subsection{Vertical-Axis Wind Turbine Cluster Design: Aerodynamic Considerations}

In order to investigate the characteristics of the proposed triangular cluster design, we conduct a suite of large-eddy simulations in a computational domain containing three of the same 
turbines defined in Table 1. The basic domain size is $L_{x} \times L_{y} \times L_{z}=72 \mathrm{~m} \times 48 \mathrm{~m} \times 25 \mathrm{~m}=$ $60 \times 40 \times 20.8 D$ and is spanned by $N_{x} \times N_{y} \times N_{z}=288 \times 192 \times 192$ grid nodes. At such resolution $(\mathrm{d} x=\mathrm{d} y=0.25 \mathrm{~m})$, the turbine diameter is covered by about $5 \times 5$ horizontal grid points, which is similar to the validation runs. These remain constant for the analyses used in this sub-section (except for the domain-size sensitivity analysis detailed later). The power coefficient $C_{P}$ of a single isolated turbine, as modelled by the LES, is 0.36 . Since the wake deficit increases with $D$ and decreases with the distance between the turbines $L, L / D$ emerges as an important dimensionless number to consider; that is, in addition to its impact on the cumulative shadowing angle $\beta$ illustrated in Fig. 6 and discussed above. In order to investigate the optimal distance, various $L / D$ ratios ranging from 2 to 8 were simulated.

Before conducting these simulations however, the computational set-up needed verification; therefore, for a fixed value of $L / D=6$, an analysis of the sensitivity of the results to the domain size was performed (such that the domain size and number of grid points increase proportionally, and thus the grid resolution is unchanged). Two parameters were investigated for sensitivity to the domain size: the cluster-averaged power coefficient $C_{P}$ and the wake velocity deficit at $15 \mathrm{D}$ and $20 \mathrm{D}$ downstream of the cluster. The wake velocity deficit is averaged in time and over a $y-z$ rectangle that is aligned in the $x$-direction with the turbine cross-section projected area. As can be seen from Fig. 7, if one compares the $80 \times 27,80 \times 40$, and $80 \times$ 54 runs, changes in domain width $L_{y}$ can be significant when $L_{y}$ becomes very small $(27 D)$. For such narrow domains, the cross-flow area blocked by the turbines becomes large and prevents correct sideways deflection of the streamlines. Since our domain is periodic in $y$, a small $L_{y}$ allows the clusters to interact with "virtual" adjacent ones. The figure suggests that a minimal $L_{y} \geq 40 D$ should be used since increasing the transverse domain size beyond that, to $L_{y}=54 D$, results in insignificant changes in the average $C_{P}$ or in wake recovery.

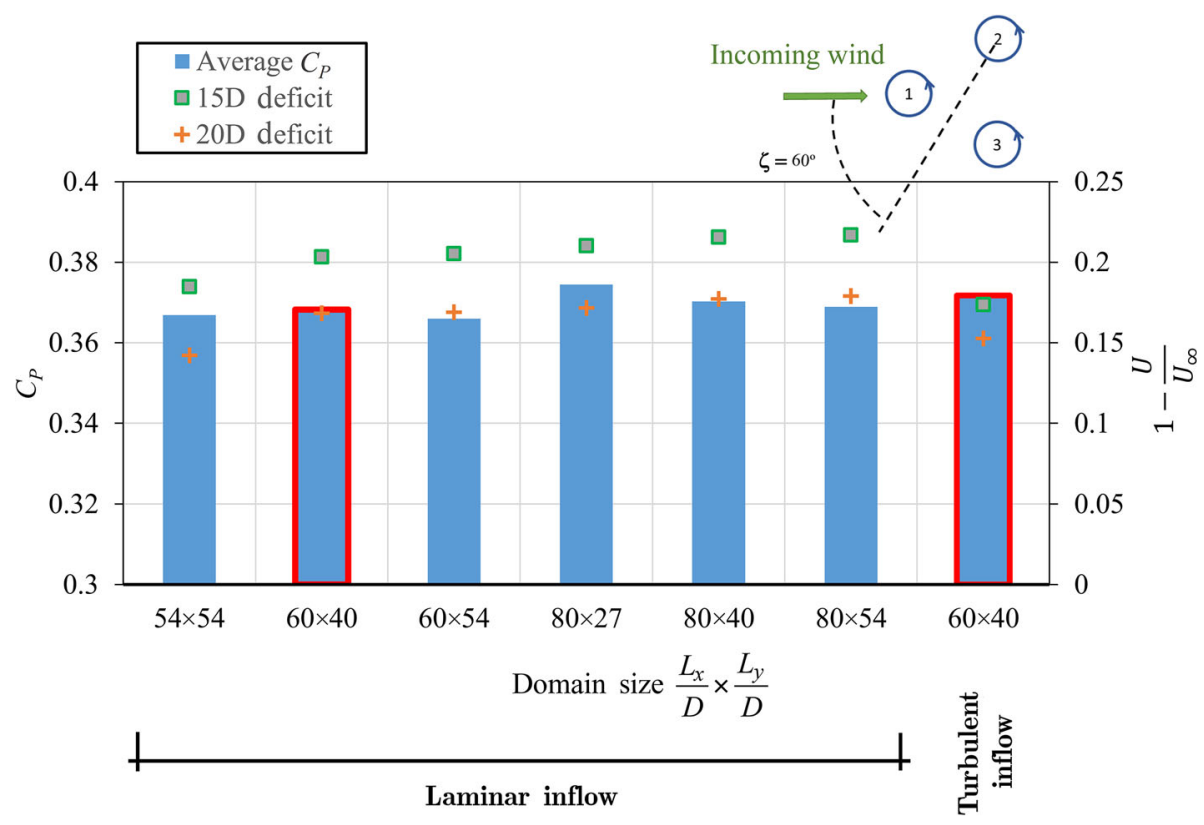

Fig. 7 Domain size sensitivity analysis. The adopted size is $60 D \times 40 D . C_{P}=$ Turbine power/ $\left(0.5 \rho A U_{\infty}^{3}\right)$, where $A$ is the rotor area 


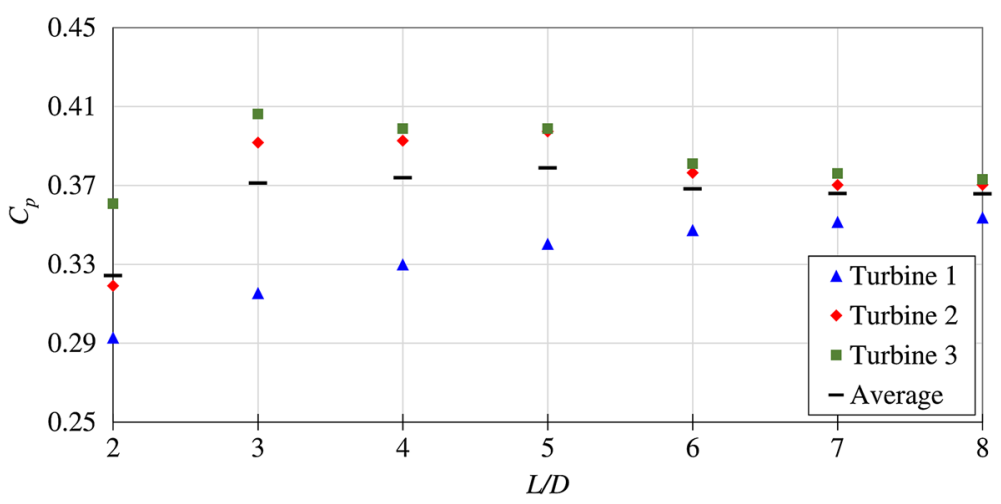

Fig. $8 C_{P}$ for each turbine in the cluster, and the average for the whole cluster, as a function of $L / D$

Changes in domain length $L_{x}$ have little impact on the average $C_{P}$ (compare the $54 \times 54$, $60 \times 54$, and $80 \times 54$ runs). However, the velocity deficit is clearly influenced by $L_{x}$ (due to downstream boundary-condition effects). Based on the sensitivity analysis results in Fig. 7, a minimal $L_{x}=40 D$ was deemed necessary (and sufficient) to avoid an impact of the domain length on the average $C_{P}$ and the wake velocity deficit (compare the $60 \times 40$ to the $60 \times 54$, or the $80 \times 40$ to the $80 \times 54$ runs). Therefore, a domain size of $L_{x}=60 D$ by $L_{y}=40 D$ is adopted for the single-cluster simulations hereafter. All these simulations were conducted using an imposed laminar logarithmic-profile inflow with a surface roughness length $z_{0}=0.001 \mathrm{~m}$ and a friction velocity $=0.5 \mathrm{~m} \mathrm{~s}^{-1}$. To assess the influence of the turbulence levels in the inflow, a simulation was conducted using inflow planes from a precursor periodic turbulent run. As can be seen in Fig. 7, using a turbulent inflow reduces the deficit values at $15 \mathrm{D}$ and $20 D$ downstream of clusters significantly, which is expected since the increase in turbulence intensity increases momentum entrainment into the wake and speeds up its recovery.

With the basic domain size set, simulations with triangular clusters for different $L / D$ ratios were conducted using, at first, the unique wind direction of $60^{\circ}$ depicted in Fig. 7. Based on simulation results for different cases (see Fig. 8), it is obvious that increasing $L / D$ improves the performance of the first (upwind) turbine due to the decrease in upstream blockage effect from turbines 2 and 3. Due to the rotation direction of the first turbine (shown in Fig. 7), which deflects the flow towards the third turbine, the third turbine has a slightly higher $C_{P}$ value compared to the second turbine. On the other hand, Fig. 8 illustrates that the performance of the second and third turbines first improves as $L / D$ increases from 2 to 3 , then reaches a plateau until $L / D=5$, and finally decreases again. When $L / D>5$, these turbines are less able to utilize the higher wind speed induced from the flow deflection by the upstream rotor. The cluster-averaged $C_{P}$ value (related to the upstream wind speed $U_{\infty}$ ) thus peaks at an intermediate $L / D$ value. The three cases with the highest average $C_{P}$ value, corresponding to $L / D$ values of 3,4 and 5 , were hence selected for further analysis.

These analyses consisted of simulations where all parameters remain the same for a given $L / D$, but with a different incoming flow orientation. We aim to investigate the omnidirectionality of the proposed VAWT clusters, as well as to find the most efficient VAWT spacing averaged over all wind directions. Figure 9a shows the average $C_{P}$ value versus incoming wind direction; the case with $L / D=5$ has the highest $C_{P}$ averaged over all turbines for all wind directions. This is confirmed in Fig. 9b that depicts the influence of $L / D$ on $C_{P}$ averaged over all wind directions and all turbines, and normalized by the $C_{P}$ value of a single 

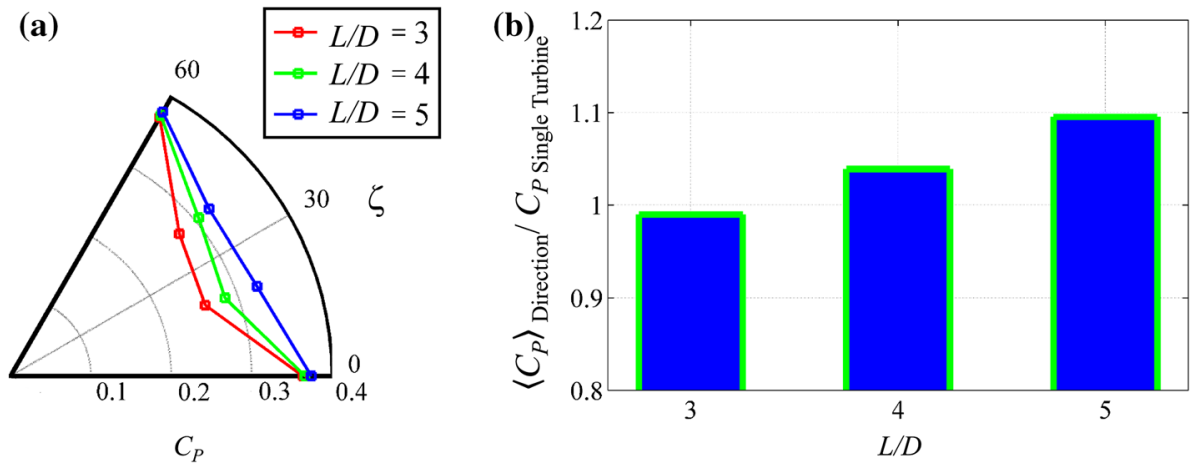

Fig. 9 a Triangular cluster-average $C_{P}$ versus wind direction $\zeta$. b Cluster-averaged $C_{P}$, also averaged over all wind directions, and normalized by the $C_{P}$ of a single isolated turbine (angled brackets denote averaging)

isolated turbine. The set-up with $L / D=5$ has improved performance because the angle at which the vertical-axis wind turbines cast shadows on downstream turbines $(\beta)$ is reduced due increasing distance between turbines, as well as because wake recovery is improved when shadowing occurs at the larger recovery distance. Finally, a key observation from Fig. $9 \mathrm{~b}$ is that the average $C_{P}$ is about $10 \%$ higher than that for a single isolated turbine when $L / D=$ 5 ; this confirms our premise that the synergistic interaction between closely-spaced turbines can indeed result in a higher overall power generation when exploited adequately.

\subsection{Farm Design: Cluster Wake and Recovery Considerations}

With an effective cluster design selected, we now turn our attention to the design of farms based on these clusters. An important parameter in designing and optimizing wind farms is the distance required for wind speed and power recovery downstream of turbines (Hezaveh et al. 2016). This applies for arrays consisting of individual turbines, as well as of clusters (unless there is a dominant known wind direction, which is not an assumption made here). The wind-speed deficit $\left(1-U(x, y, z) / U_{\infty}(z)\right)$ was averaged over the $y-z$ planes encompassing the whole cluster (projected flow-normal area) at varying $x$ distances from the hub using data from the same simulations described in the previous sub-section. We also investigated various values of $L / D$ to confirm that our choice of $L / D=5$, made based on the power output of an isolated cluster, does not produce longer wakes than for other $L / D$ values. The results reported in Fig. 10 indicate that increasing the distance between the turbines in each triangle cluster significantly reduces the distance needed for the wind speed to recover to $75 \%$ of its upstream value $U_{\infty}$. It is clear from the figure that the recovery distance to $75 \%$ speed is reduced from $25 D$ for $L / D=3$ to $15 D$ for $L / D=5$. The choice of the $75 \%$ recovery speed is somewhat arbitrary, and other thresholds can be selected, of course. However, the comparative analysis of the recovery distances would reach the same conclusions regarding the optimal $L / D$ to adopt, irrespective of the exact recovery threshold.

Recovery is an important criterion for designing a wind farm, which further confirms the selection of $L / D=5$. In a wind farm, it is important that downstream turbines are placed at distances were the available flow has recovered to significant levels of its upstream undisturbed speed (e.g. to over $75 \%$, although higher levels are advantageous) so that the power-generation capacity of these turbines is not under-utilized. Furthermore, as shown in Fig. 10, by increasing the distance between the turbines in the clusters, the effect of incoming wind direction 

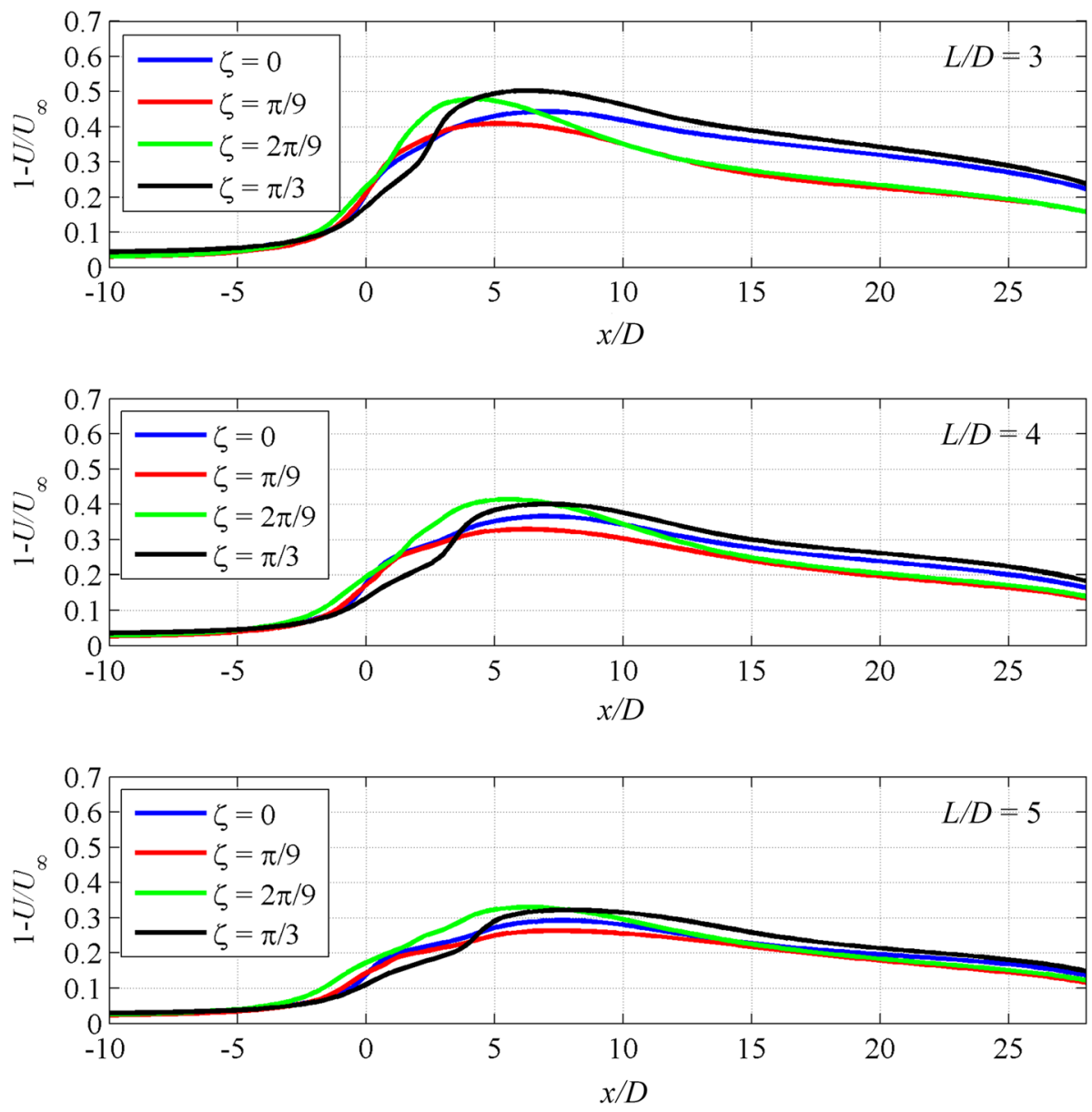

Fig. 10 Comparison between averaged velocity deficit for various $L / D$ and different wind directions $\zeta$

on the recovery distance is reduced. The $L / D=3$ recovery is sensitive to the change in incoming wind direction $\zeta$; the recovery distance to $75 \%$ of upstream flow speed occurs anywhere between $18 D$ and $28 D$ as the wind direction changes. The recovery for $L / D=5$ on the other hand is much less sensitive to wind direction and thus yields more omni-directional farms. The results also indicate that when designing farms based on clusters with $L / D=5$, the velocity recovery for a separation of $10 D$ between the clusters is about $70-75 \%$, while a separation of $20 \mathrm{D}$ allows a recovery to over $80 \%$ of upstream flow speed. Both of these separations are tested in the full-farm simulations below. While other separations can be examined, the outcome of the testing of our hypothesis regarding the potential benefits from synergistic interaction between vertical-axis wind turbines remains the same.

\subsection{Farm Design: Performance Assessment}

Now we tackle the main question: can synergistic interactions between vertical-axis wind turbines increase wind-farm power density? Practically, we need to investigate whether farms 
(a)
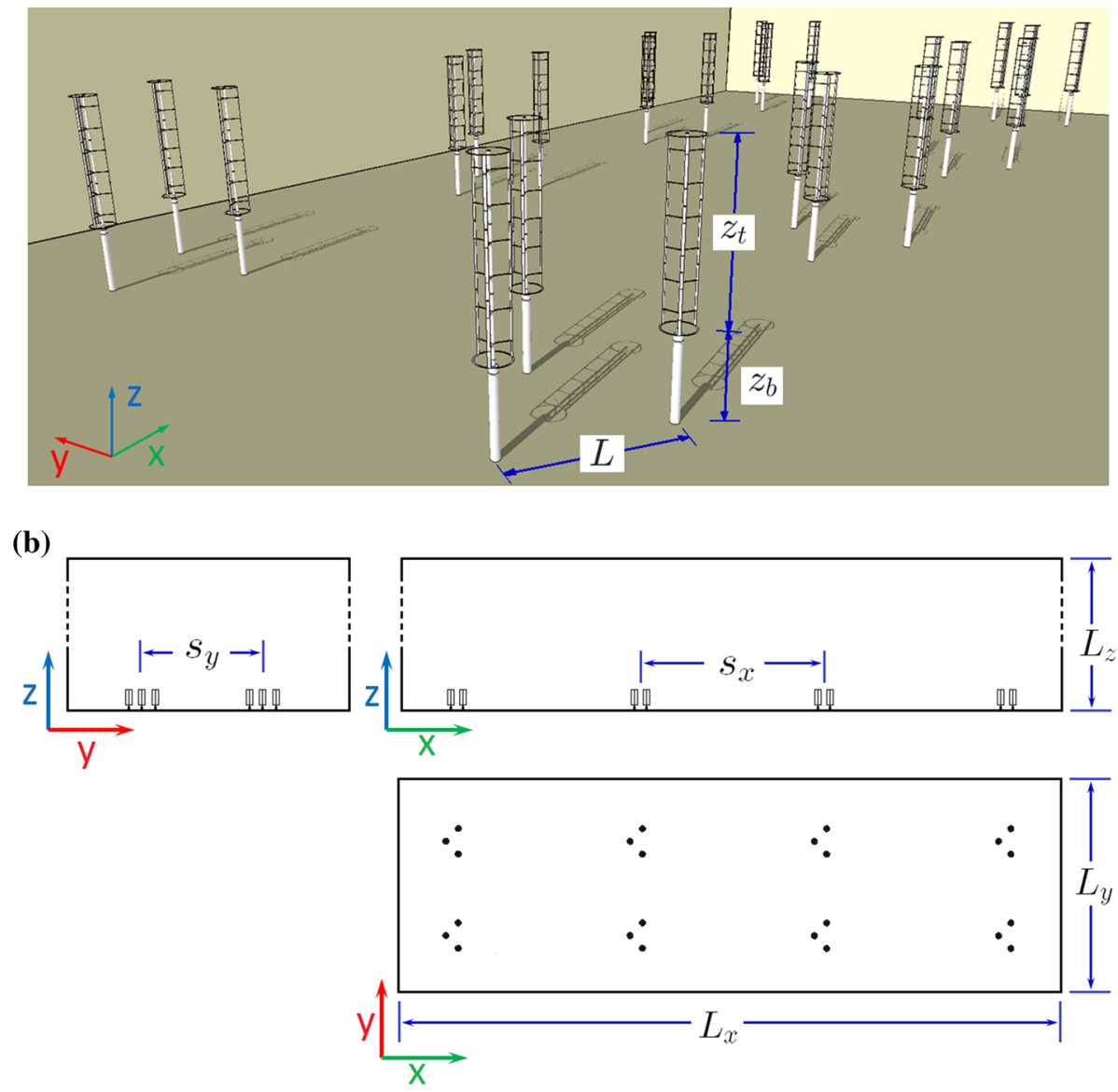

Fig. 11 Schematic of the wind-farm configuration with the VAWT triangular clusters, with turbine-to-turbine separation distances of $L=5 D$ and inter-cluster spacing of $20 D$ for an aligned cluster configuration

with synergistic clusters have improved performance (produce more power per unit land or per unit invested cost) relative to two prototypical layouts of wind farm, aligned and staggered regular arrays. Based on the size of the selected turbine and the results obtained above, eleven farm configurations were simulated. One configuration is illustrated in Fig. 11, while four more layouts can be visualized in the streamwise velocity plots of Fig. 12; the turbines are the same as those detailed in previous sections. The simulations used are all periodic (representing an infinite farm), with $N_{x} \times N_{y} \times N_{z}=320 \times 160 \times 336$ nodes and $L_{x} \times L_{y} \times L_{z}=96 \mathrm{~m} \times$ $48 \mathrm{~m} \times 32 \mathrm{~m}$. The resolution yields $4 \times 4$ horizontal grid nodes per rotor diameter, which is comparable to the validation tests presented earlier. The vertical height of the simulation domain is selected based on a sensitivity analysis performed for the $10 \mathrm{D}$ staggered-spacing wind farm. Domains with vertical heights of 32, 45 and $54 \mathrm{~m}$ were chosen and the total power coefficients (we use two definitions, $C_{P}$ and $C_{P}^{*}$, that are described below) of these three domains are compared. Due to the small blockage ratio of the wind turbines (projected area of turbines normal to the flow over the $y-z$ area of the computational domain), which 

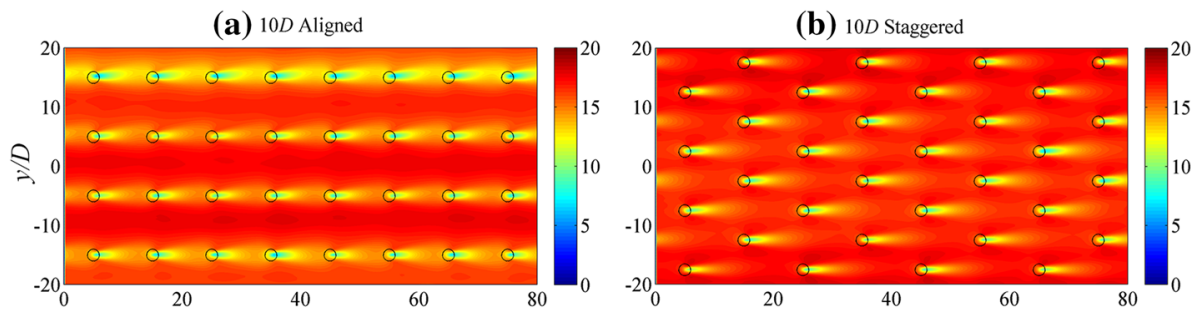

(c) $10 \mathrm{D}$ Cluster Staggered, $0^{\circ}$

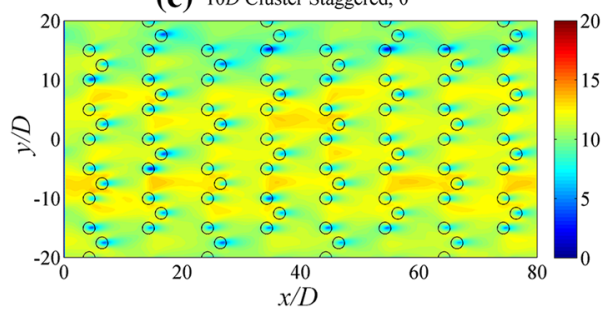

(d) $10 \mathrm{D}$ Cluster Staggered, $60^{\circ}$

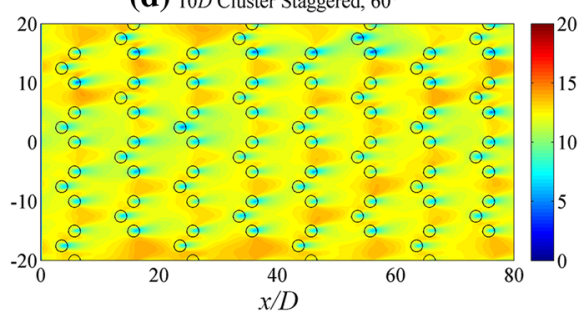

Fig. 12 Streamwise velocity magnitude in wind farms with $10 \mathrm{D}$ horizontal spacings, mean flow from left to right: a regular aligned, $\mathbf{b}$ regular staggered, $\mathbf{c}$ cluster staggered with $0^{\circ}$ wind direction, and $\mathbf{d}$ cluster staggered with $60^{\circ}$ wind direction

Table 2 Vertical height of the computational domain versus farm-averaged power coefficient for a wind farm with $10 \mathrm{D}$ staggered configuration

\begin{tabular}{llll}
\hline & \multicolumn{3}{l}{ Vertical domain height } \\
\cline { 2 - 4 } & $32 \mathrm{~m}$ & $45 \mathrm{~m}$ & $54 \mathrm{~m}$ \\
\hline$C_{P}$ & 0.45 & 0.46 & 0.46 \\
$C_{P}^{*}$ & 0.52 & 0.53 & 0.53 \\
\hline
\end{tabular}

is $<3.75 \%$ in our runs, the variation of the total wind-farm-averaged power coefficients with domain height $<1 \%$ (Table 2). Therefore, the 32-m high domain was deemed sufficient and chosen accordingly; this agrees with Sarlak et al. (2016) concerning appropriate blockage ratios for wind-energy studies.

Six of the simulated cases are prototypical wind farms with staggered or regular array configurations and with separation distances of $5 D, 10 D$ or $20 D$; these configurations result in 128,32 and 8 turbines in the computation domain, respectively. Four additional experiments were conducted using staggered clusters (with $L / D=5$ separation within each cluster) with wind direction of $0^{\circ}$ or $60^{\circ}$ and with inter-cluster distances of $20 \mathrm{D}$ or $10 \mathrm{D}$ for each direction, corresponding to 24 and 96 turbines in the domain, respectively. It should be mentioned that since the LES model uses periodic boundary conditions in both $x$ and $y$ directions, these simulations correspond to infinite wind farms with an infinite number of turbines. Therefore, the number of turbines in the computational domain does not influence the results when normalized per turbine. Finally, one experiment is conducted using $20 \mathrm{D}$ spacing and aligned clusters with a $60^{\circ}$ wind direction relative to the cluster. To visualize the differences in the flow patterns in these designs, the average streamwise velocity component for a few selected configurations is shown in Fig. 12. The lower values in the staggered configurations reflects the higher power extraction resulting from a larger number of turbines, and therefore metrics that allow consistent comparison of these configurations are needed. Finding such metrics 


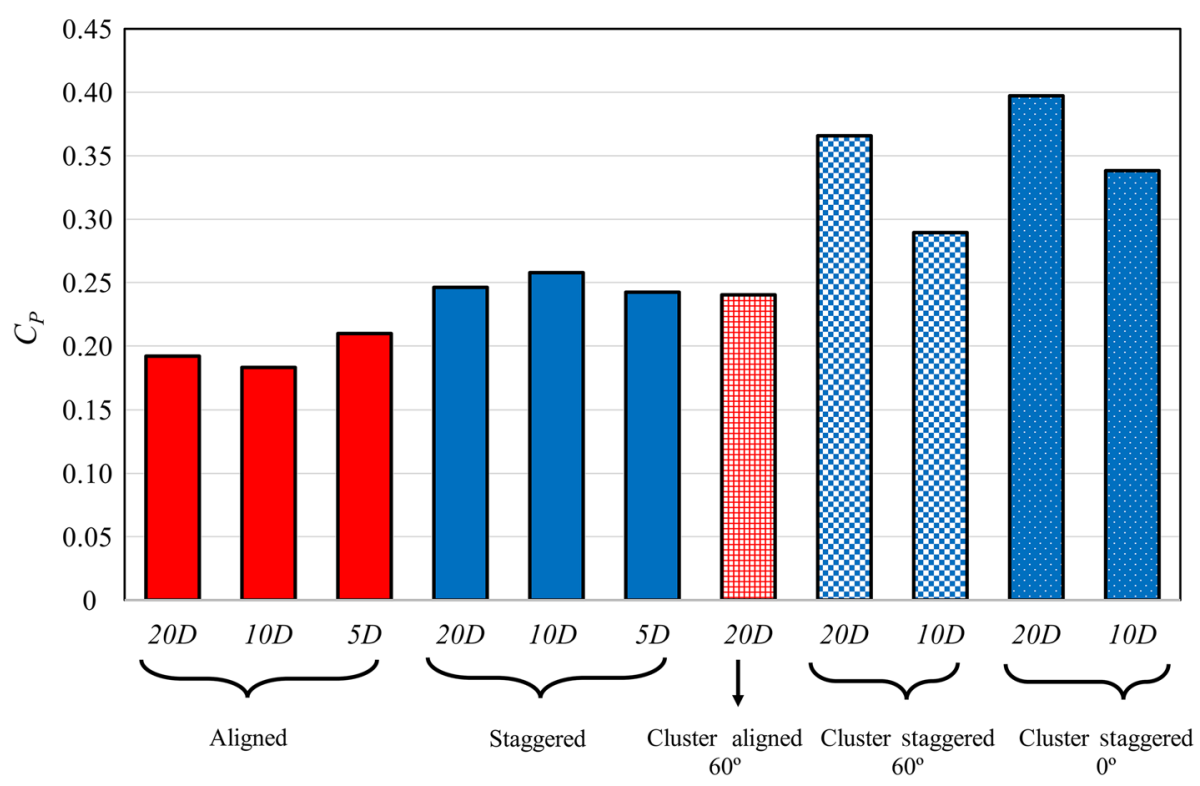

Fig. 13 Average wind-farm $C_{P}$ values for various configurations (staggered or aligned, clustered or regular) and for various separation distances

is not straightforward as discussed in Meyers and Meneveau (2010) and Goit and Meyers (2015).

The most direct metric and the easiest to compute is the average wind-farm $C_{P}$ value that uses as a reference speed the average streamwise velocity component in the whole windfarm volume containing the vertical-axis wind turbines (i.e. over a volume spanning a full $x-y$ plane and the $z$ domain from the bottom to the top of the blades). The comparison of this $C_{P}$ value for different layouts is shown in Fig. 13. As anticipated the staggered cases have higher $C_{P}$ values compared to the aligned ones, for both the clustered and regular designs. Of more interest and relevance is that the clustered designs consistently produce higher power than the prototypical design for any spacing. As indicated previously, the $C_{P}$ value for an isolated turbine is 0.36 and the cluster-staggered designs with an inter-cluster spacing of $20 D$ surpass this value over the whole wind farm for both wind directions. This is due to the gain in average $C_{P}$ that clusters allow, and the large inter-cluster spacing that minimizes the effects of being in the wake of the upstream cluster. By reducing the distance between clusters to $10 D$, the average $C_{P}$ value decreases but remains significantly higher than for the corresponding regular wind farms.

Another important result is that the staggered configurations, even at small separation distances, consistently perform better than the aligned ones. The streamwise separation in the staggered $10 D$ case, for example, is the same as the separation in the $20 D$ aligned case, and yet the staggered $10 D$ layout yields a higher $C_{P}$. One physical reason for this improved performance is that in the staggered cluster farms, in addition to the synergistic interactions within each cluster, the clusters themselves probably interact favourably. One can observe, for example, in Fig. 12c, d that two adjacent clusters produce flow acceleration in between clusters, allowing the next staggered row to benefit from this flow deviation. This is exactly similar to the acceleration within a cluster but now occurs in between clusters, suggesting a 
fractal attribute to these synergistic interactions (although with only two fractal generations here).

The results in Fig. 13, however, exclude an important difference between these periodic simulations. Due to higher drag forces exerted on the ABL in cases with higher densities $(5 D$ spacing) or cases with more efficient farm layouts, the required pressure gradient imposed in the simulations to yield a steady-state mean flow will also be higher. In the LES, at each timestep, the drag exerted on the ABL by the whole wind farm and the ground surface is computed and the needed mean streamwise pressure gradient to balance this drag is imposed. This gradient eventually reaches a steady state as the mean flow equilibrates. As a result, the different cases have unique pressure gradients over the wind farm, and the supplied power input (estimated as the product of the pressure gradient force and the streamwise velocity magnitude) into the domain is not consistent across all cases. As found in Meyers and Meneveau (2010), Goit and Meyers (2015), several approaches can be used to overcome this potential inconsistency. Since our simulations omit the Coriolis force, the best approach is to normalize the power extracted by the power supplied in the wind-farm volume to the simulations. With no Coriolis force and under steady-state conditions, the pressure gradient force has to balance the total (turbine + ground surface) drag. One can thus characterize these two equal and opposite forces with a squared friction velocity related to the total domain drag $u_{\tau H}$ (defined similarly to Calaf et al. 2010; Goit and Meyers 2015). The total power input is thus proportional to $U_{T} u_{\tau H}^{2}$, where $U_{T}$ is the streamwise velocity component averaged over the wind-farm volume (average of the domain containing the blades as defined before). In large wind farms, this is the rate of mean kinetic energy input into the domain that can be extracted by the turbines. The velocity upwind of a specific row (used to define $C_{P}$ before) is an outcome of this input rather than the main source of energy as in very small farms. Thus, the kinetic energy that can be extracted in large wind farms scales with the pressure drop and with the farm-volume-averaged streamwise velocity component $U_{T}$, and since these farms influence the atmospheric pressure field as well as flow inside them significantly, they influence the power available to them. Therefore, in order to be able to compare the various configurations without this pressure-drop discrepancy, the following $C_{P}^{*}$ relation normalized per unit power input, is introduced,

$$
\begin{gathered}
C_{P}^{*}=\frac{P_{T}}{\frac{1}{2} \rho A\left(u_{\tau H}\right)^{2} U_{T}}, \\
u_{\tau H}^{2}=\Delta P_{\text {drop }} \frac{L_{x}}{L_{z}},
\end{gathered}
$$

where $P_{T}$ is the average power per turbine, $u_{\tau H}$ is the root square of the total mean drag (on ground + turbines), which scales with the total pressure drop $\Delta P_{d r o p}$ across the farm, and $A$ is the rotor area of a single turbine. The comparison of this new performance metric for the various configurations is presented in Fig. 14. Since $u_{\tau H}$ is roughly about $10 \%$ of the wind speed, $C_{P}^{*}$ is about $100 C_{P}$ and should not be interpreted in the same way as the classic power coefficient. Even after normalizing the total power generated in these layouts by the power input for each case, the cluster cases maintain the highest $C_{P}^{*}$ value, implying that these cases are able to extract more energy from the applied pressure gradient in the field compared to regular wind farms. The relative differences in the performance of wind farms are expected to be closer to the differences depicted in Fig. 13 for smaller farms $\left(C_{P}\right.$ is strictly applicable only for a single row), and closer to those in Fig. 14 for larger farms.

A comparison of the power density per unit land area used for the various configurations has also been performed, confirming that clustered designs increase the power density, and 


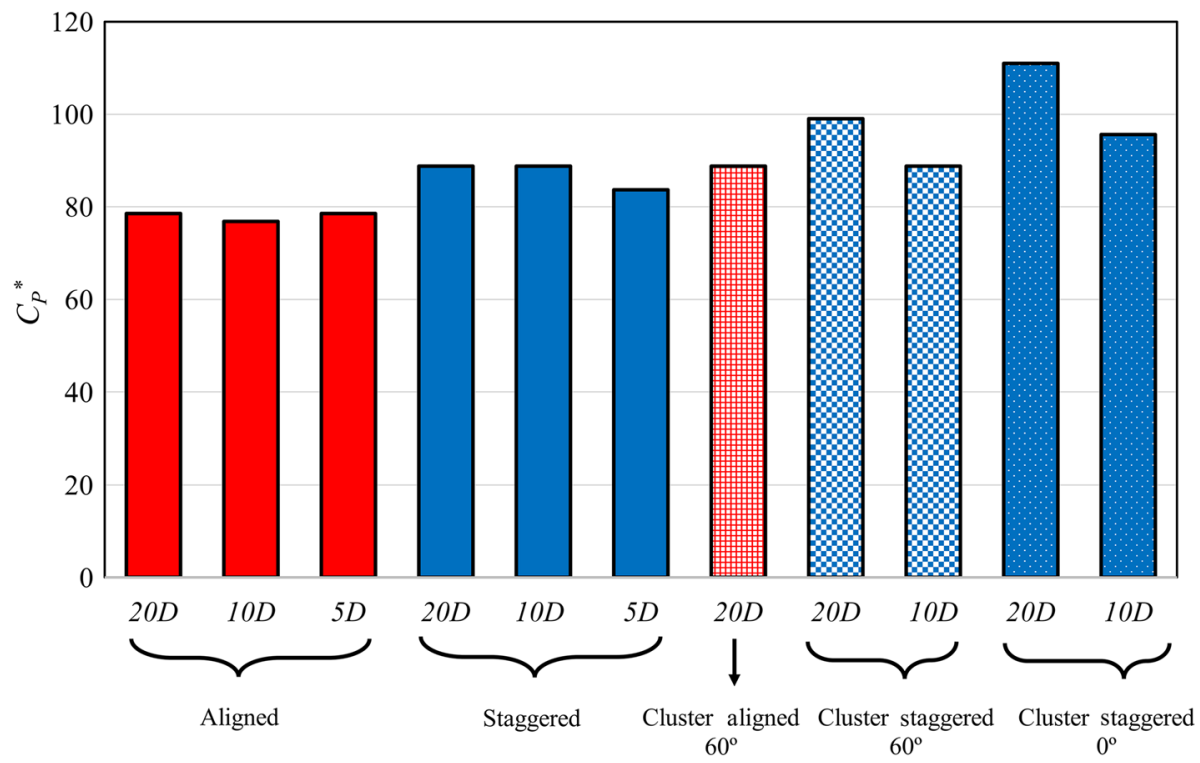

Fig. 14 Average wind-farm $C_{P}^{*}$ values normalized per unit applied power input to the turbine domain for various configurations (staggered or aligned, clustered or regular) and for various separation distances

validating our hypothesis. However, the results have the caveat that the power density is invariably higher for smaller spacings, even when the turbines in the farm are not being used efficiently (low $C_{P}$ ). Therefore, power density itself cannot be used as a metric for optimizing farm layout. In order to have a more realistic and practical metric, the total capital cost per unit power generation $T_{\text {total }}$ is computed. Since the power generation for each farm is proportional to the sum of the $C_{P}^{*}$ values of all the individual turbines in a given lot area of fixed size, we use this sum, denoted as $C_{\Sigma P}^{*}$, for normalization instead of the actual power. The capital costs consist mainly of the cost of the land and the turbines. The following normalized energy cost function can thus be computed (similar to Meyers and Meneveau 2012)

$$
\frac{T_{\text {total }}}{C_{\Sigma P}^{*}}=\left(\frac{T_{\text {land }} A_{L}+\Gamma_{A} A_{L} T_{\text {turbine }}}{C_{\Sigma P}^{*}}\right)=\left(\frac{T_{\text {land }}}{T_{\text {turbine }}}+\Gamma_{A}\right) \frac{A_{L} T_{\text {turbine }}}{C_{\Sigma P}^{*}},
$$

where $\Gamma_{A}$ is the wind turbine density per unit area and $A_{\mathrm{L}}$ is the total lot land area. $T_{\text {land }}$ is the cost of land per unit area and $T_{\text {turbine }}$ is the cost of a single turbine. Using different land-cost to turbine-cost ratios, and the cost for a typical individual turbine similar to the one simulated $(\approx$ \$US 10,000) (Dabiri 2011) in Eq. 11 , the normalized energy costs were computed and plotted in Fig. 15. Using this comparison metric also indicates that the triangular-cluster staggered layout has the lowest capital cost per projected unit power generated, and is therefore the optimum design among those investigated.

A similar analysis has been made using total $C_{P}$ and the results also indicate that wind farms with cluster designs are the most optimal amongst those investigated here. Again, we reiterate that the comparison with $C_{P}$ is more relevant for very small farms, while if one uses $C_{P}^{*}$, the results are more representative of large farms. 


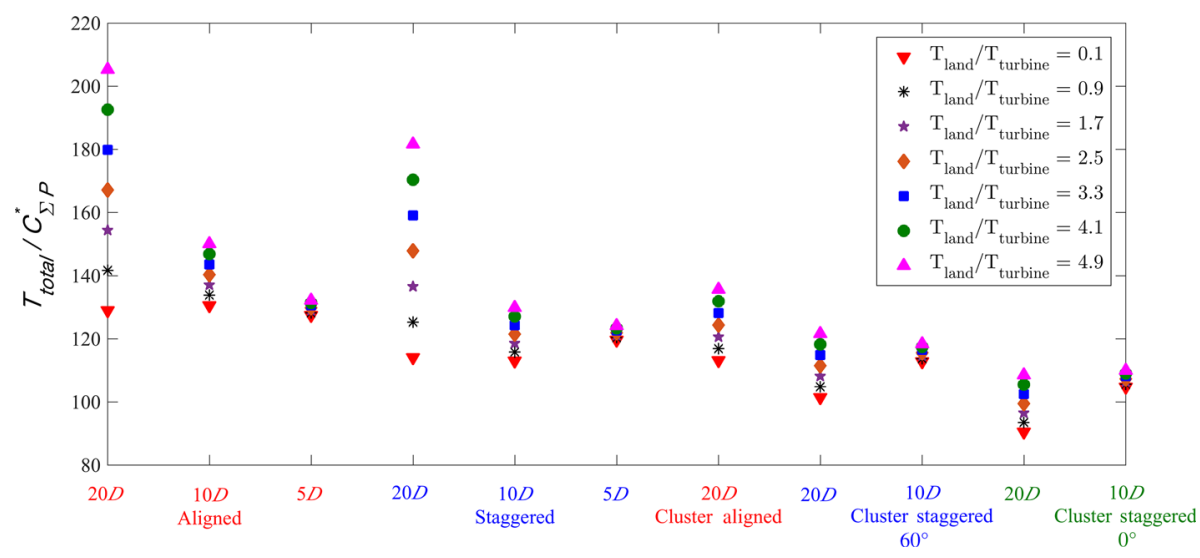

Fig. 15 Total capital cost per "unit power" generated for the various cases

\section{Conclusions}

We have presented a novel concept for optimizing the layout of large vertical-axis windturbine farms, taking advantage of synergistic interactions between closely-spaced turbines that were previously shown to yield higher power for a limited number of turbines. Using an actuator-line-model representation of the turbines, embedded in a large-eddy simulation, the modelled wake generated by two counter-rotating turbines is first successfully validated against observations from field experiments. To take advantage of the high wind speed created by the flow deflection of vertical-axis wind turbines when placed in close vicinity, we propose a triangular cluster design consisting of three vertical-axis wind turbines, which form the basis for larger wind farms. The triangular design is the one that best exploits flow acceleration, with a minimal increase in wake shadowing.

The influence of inter-turbine spacing relative to their diameter, $L / D$, was then investigated to optimize a single cluster in terms of the total generated power, the omni-directionality of its performance, and the needed downstream wake-recovery distance. Changing the turbine spacing, the cases with $L / D$ values of 3, 4 and 5 were shown to generate the highest clusteraveraged power. Further tests were then performed with these three spacings only, and the case with $L / D=5$ emerged as the one with the highest cluster-averaged power over all wind directions: the generated power for this case is about $10 \%$ higher than that produced by three isolated turbines. Furthermore, $L / D=5$ results in the lowest variation of the generated power with wind direction, and the downstream wake-recovery distance is the shortest (since the cluster is more "porous"). Therefore, this cluster design confirmed the use of synergistic vertical-axis wind-turbine interactions to increase power production, and would generate a higher power density (power generated per unit land used) due to the proximity of the rotors. It was hence adopted for configuring large VAWT wind farms.

Farms that use this advanced cluster design, and a sufficient distance for wake recovery between clusters of $10 \mathrm{D}$ and $20 \mathrm{D}$, were then compared to prototypical aligned and staggered configurations for infinitely-large wind farms, with different turbine horizontal spacings of $5 D, 10 D$ and $20 D$. For the very large wind farms chosen, the results show that the average wind-farm power coefficient, using two distinct normalizations, is much higher for the staggered-triangle clusters than for the wind farms with regular configurations. Using these average power coefficient results and a simple capital cost function for the whole wind farm, 
while varying the land-to-turbine cost ratio, we also showed that the wind-farm design with staggered-triangle clusters is the optimal design (amongst the ones considered here) in terms of cost per unit power produced.

These results strongly indicate that VAWT farms can and should be configured using different approaches than those used for horizontal-axis rotors (although the potential benefits of HAWT clustering could also be investigated). A significant increase in power and decrease in capital costs can be achieved using the ability of vertical-axis wind turbines to positively boost the power production of nearby turbines if properly configured. A further important aspect of the results is that, in addition to turbine interactions within a cluster, the clusters themselves interact synergistically, further boosting power production. It should also be mentioned that one of our criteria in optimizing the clusters and farms was omnidirectionality. We sought to propose configurations with performances that are not strongly dependent on wind direction since this is also a major advantage of individual vertical-axis wind turbines. However, if this criterion is relaxed, for example in places where there is a dominant wind direction, the optimal cluster designs can be very different and can use this synergistic interaction between clusters as well, with potentially higher power densities.

Finally, one factor that plays an important role in modulating power output from a large wind farm is atmospheric stability. It has been shown that the diurnal cycle and a range of ABL stability strongly influence the performance of large HAWT wind farms (Lu and PortéAgel 2011; Abkar et al. 2016), and the same is expected for the VAWT farms used herein. However, we focused on the basic neutral case only, and ALM-LES model investigations of the effect of atmospheric stability on VAWT wind-farm operation are left for future studies.

Acknowledgements This work was supported by the Siebel Energy Challenge and the Andlinger Centre for Energy and the Environment of Princeton University. The simulations were performed on the supercomputing clusters of the National Centre for Atmospheric Research through project P36861020 and UPRI0007, and of Princeton University.

Open Access This article is distributed under the terms of the Creative Commons Attribution 4.0 International License (http://creativecommons.org/licenses/by/4.0/), which permits unrestricted use, distribution, and reproduction in any medium, provided you give appropriate credit to the original author(s) and the source, provide a link to the Creative Commons license, and indicate if changes were made.

\section{Appendix A: Field Experimental Set-Up}

Experiments were conducted at the Caltech Field Laboratory located in the Antelope valley of northern Los Angeles County in California, USA (Kinzel et al. 2015). The surroundings are flat desert terrain with no obstacles for at least $1.5 \mathrm{~km}$ horizontally in all directions. The turbines were in operation, and data were collected, mostly in the middle of the day when the ABL was not neutrally stable. Therefore, buoyancy generation contributes to the turbulence levels of the flow. For the validation, periods with near-neutral stability were identified and used. The turbines are rated at $1.2 \mathrm{~kW}$ (Windspire Energy, Inc., Reno, Nevada, USA), with a total height of $9.1 \mathrm{~m}$, a rotor height of $6.1 \mathrm{~m}$, and a diameter of $1.2 \mathrm{~m}$. Their maximum rotation rate is 420 revolutions $\mathrm{min}^{-1}$ at a wind speed of $12 \mathrm{~m} \mathrm{~s}^{-1}$, yielding a tip-speed ratio $\lambda=2.3$. The cut-in and cut-out speeds for this turbine are 3.8 and $12 \mathrm{~m} \mathrm{~s}^{-1}$, respectively.

The wind velocity was measured from a movable 10-m high meteorological tower (Aluma Towers, Inc., Vero Beach, Florida, USA) with seven, vertically-staggered, three-component ultrasonic anemometers (Campbell Scientific CSAT3, Logan, Utah, USA). The sensors are equally spaced by $1 \mathrm{~m}$ between the top and the bottom of the turbine rotor, i.e., between 
heights of 3 and $9 \mathrm{~m}$. The data collection frequency was $10 \mathrm{~Hz}$ and the tower was moved to the various horizontal locations where data were to be collected.

The velocity profiles were measured at several locations along the centreline of the turbine arrays. For the configuration shown herein, these positions were $15 D$ and $1.5 D$ upstream, as well as $2 D$ and $8 D$ downstream, from the front of the array. The measurements were taken for at least $150 \mathrm{~h}$ at each position. The dataset was filtered for times when the freestream wind speed was within the cut-in and cut-out wind speeds of the turbines and the wind direction was within $\pm 10^{\circ}$ from the array centreline. The mean horizontal wind speed as measured by the reference sensor was $8.2 \mathrm{~m} \mathrm{~s}^{-1}$ during the times when the doublet configurations used here were tested, leading to an average Reynolds number of $\approx 106$ based on rotor diameter. The prevailing wind direction was south-south-west, i.e., $223^{\circ}$ for doublet configurations.

\section{References}

Abkar M, Sharifi A, Porté-Agel F (2016) Wake flow variability in a wind farm throughout a diurnal cycle. J Turbul 17:420-441. https://doi.org/10.1080/14685248.2015.1127379

Araya DB, Craig AE, Kinzel M, Dabiri JO (2014) Low-order modeling of wind farm aerodynamics using leaky Rankine bodies. J Renew Sustain Energy 6:63118. https://doi.org/10.1063/1.4905127

Bou-Zeid E, Meneveau C, Parlange M (2005) A scale-dependent Lagrangian dynamic model for large eddy simulation of complex turbulent flows. Phys Fluids 17:25105. https://doi.org/10.1063/1.1839152

Bou-Zeid E, Parlange MB, Meneveau C (2007) On the parameterization of surface roughness at regional scales. J Atmos Sci 64:216-227. https://doi.org/10.1175/JAS3826.1

Calaf M, Meneveau C, Meyers J (2010) Large eddy simulation study of fully developed wind-turbine array boundary layers. Phys Fluids 22:1-16. https://doi.org/10.1063/1.862466

Calaf M, Higgins C, Parlange MB (2014) Large wind farms and the scalar flux over an heterogeneously rough land surface. Boundary-Layer Meteorol 153:471-495. https://doi.org/10.1007/s10546-014-9959-6

Chamorro LP, Porté-Agel F (2009) A wind-tunnel investigation of wind-turbine wakes: boundary-layer turbulence effects. Boundary-Layer Meteorol 132:129-149. https://doi.org/10.1007/s10546-009-9380-8

Chamorro LP, Porté-Agel F (2010) Effects of thermal stability and incoming boundary-layer flow characteristics on wind-turbine wakes: a wind-tunnel study. Boundary-Layer Meteorol 136:515-533. https://doi. org/10.1007/s10546-010-9512-1

Claessens MC (2006) The design and testing of airfoils for application in small vertical axis wind turbines. Masters Thesis, McMaster University, Hamilton, Ontario, Canada

Cortina G, Calaf M, Cal RB (2016) Distribution of mean kinetic energy around an isolated wind turbine and a characteristic wind turbine of a very large wind farm. Phys Rev Fluids 1:74402. https://doi.org/10.110 3/PhysRevFluids.1.074402

Dabiri JO (2011) Potential order-of-magnitude enhancement of wind farm power density via counter-rotating vertical-axis wind turbine arrays. J Renew Sustain Energy 3:43104. https://doi.org/10.1063/1.3608170

Goit JP, Meyers J (2015) Optimal control of energy extraction in wind-farm boundary layers. J Fluid Mech 768:5-50. https://doi.org/10.1017/jfm.2015.70

Hezaveh SH, Bou-Zeid E, Lohry MW, Martinelli L (2016) Simulation and wake analysis of a single vertical axis wind turbine. Wind Energy 20:713-730. https://doi.org/10.1002/we.2056

Huang J, Bou-Zeid E (2013) Turbulence and vertical fluxes in the stable atmospheric boundary layer. Part I: a large-eddy simulation study. J Atmos Sci 70:1513-1527. https://doi.org/10.1175/JAS-D-12-0167.1

Kinzel M, Mulligan Q, Dabiri JO (2012) Energy exchange in an array of vertical-axis wind turbines. J Turbul 13:N38. https://doi.org/10.1080/14685248.2012.712698

Kinzel M, Araya DB, Dabiri JO (2015) Turbulence in vertical axis wind turbine canopies. Phys Fluids 27:115102. https://doi.org/10.1063/1.4935111

Li Q, Bou-Zeid E, Anderson W, Grimmond S, Hultmark M (2016) Quality and reliability of LES of convective scalar transfer at high Reynolds numbers. Int J Heat Mass Transf 102:959-970. https://doi.org/10.1016/ j.ijheatmasstransfer.2016.06.093

Lu H, Porté-Agel F (2011) Large-eddy simulation of a very large wind farm in a stable atmospheric boundary layer. Phys Fluids 23:65101. https://doi.org/10.1063/1.3589857

Marquis M, Wilczak J, Ahlstrom M, Sharp J, Stern A, Smith JC, Calvert S (2011) Forecasting the wind to reach significant penetration levels of wind energy. Bull Am Meteorol Soc 92:1159-1171. https://doi.or g/10.1175/2011BAMS3033.1 
Meyers J, Meneveau C (2010) Large eddy simulations of large wind-turbine arrays in the atmospheric boundary layer. In: 48th AIAA aerospace sciences meeting, Orlando, Florida, USA, 4-7 January 2010, pp 1-10

Meyers J, Meneveau C (2012) Optimal turbine spacing in fully developed wind farm boundary layers. Wind Energy 15:305-317. https://doi.org/10.1002/we.469

Sarlak H, Nishino T, Martínez-Tossas LA, Meneveau C, Sørensen JN (2016) Assessment of blockage effects on the wake characteristics and power of wind turbines. Renew Energy 93:340-352. https://doi.org/10. 1016/j.renene.2016.01.101

Stevens RJAM, Gayme DF, Meneveau C (2014) Large eddy simulation studies of the effects of alignment and wind farm length. J Renew Sustain Energy 6:1-14. https://doi.org/10.1063/1.4869568

Troldborg N, Sørensen J (2014) A simple atmospheric boundary layer model applied to large eddy simulations of wind turbine wakes. Wind Energy 17:657-669. https://doi.org/10.1002/we.1608

U.S. Energy Information Administration (2013) International energy outlook 2013. Technical Report, U.S. Energy Information Administration. https://www.eia.gov/outlooks/ieo/pdf/0484(2013).pdf

Wu Y-T, Porté-Agel F (2012) Simulation of turbulent flow inside and above wind farms: model validation and layout effects. Boundary-Layer Meteorol 146:181-205. https://doi.org/10.1007/s10546-012-9757-y

Yu-ting WPF (2011) Large-eddy simulation of wind-turbine wakes: evaluation of turbine parametrisations. Boundary-Layer Meteorol 138:345-366. https://doi.org/10.1007/s10546-010-9569-x 